\title{
Characterization of surface properties of dry-coated anhydrous borax powders
}

\section{Süleyman Akpınar*}

Afyon Kocatepe University, Department of Material Science and Engineering, Afyonkarahisar, Turkey, ORCID ID orcid.org/0000-0002-7959-3464

\section{ARTICLE INFO}

Article history:

Received 15 January 2020

Received in revised form 06 August 2020

Accepted 10 September 2020

Available online 30 September 2020

Research Article

DOI: $10.30728 /$ boron.675261

\section{Keywords:}

Surface modification,

Dry coating,

Anhydrous borax

Stearic acid,

Characterisation.

\begin{abstract}
Dry particle coating of anhydrous borax (ANB) powders with hydrophobic stearic acid (SA) in the planetary ball mill was investigated. Two process parameters, the amounts of modifier agent and the processing periods, were selected to investigate their effects on solubility, wettability, dispersibility, and hydration properties of ANB powders. Each of the process parameters, i.e., SA amounts $(0.5,1$, and $2 \mathrm{wt} \%)$, and coating periods $(30,60$, and $120 \mathrm{~min})$, was set at three different levels. Structural changes in the dry-coated powders were characterised using X-ray diffraction (XRD) and photoelectron spectroscopy (XPS), Fouriertransform infrared spectroscopy (FT-IR), Scanning electron microscopy (SEM), and Differential thermal and thermogravimetric analysis (DTA-TG). A discrete coating at an efficiency of $68 \%$ was acquired with SA amount of $1 \mathrm{wt} \%$ at a coating period of $60 \mathrm{~min}$, and, thus, resulted in a $31.4 \%$ decrease (from $40 \%$ to $8.6 \%$ ) in water solubility of ANB. Moreover, this discrete SA coating made ANB more hydrophobic $\left(99^{\circ}\right)$ and prevented its further hydration. Furthermore, FT-IR and XPS results indicated that SA is coated over the surface of ANB via physical adsorption rather than chemical bonding. Thus, the effective dry coating could be applied to obtain surface-coated ANB, which offers controlled solubility in water-based suspensions.
\end{abstract}

\section{Introduction}

Anhydrous borax $\left(\mathrm{Na}_{2} \mathrm{~B}_{4} \mathrm{O}_{7}\right)$, which is produced via fusion of borax deca- or pentahydrate at approximately $1000{ }^{\circ} \mathrm{C}$, is an indispensable boron-containing compound of most industrial ceramic and glass products, offering both excellent glass-forming and fluxing properties [1-3]. The anhydride form of borax is preferred to other hydrous types since it has a slower and lower solubility than its aqueous forms, and it does not cause any foaming problems arising from water dehydration in high-temperature applications [2]. However, its solubility value is still high $\left(3.15 \mathrm{wt} \%\right.$ at $\left.25{ }^{\circ} \mathrm{C}\right)$ for direct use in the aqueous systems [4].

The soluble borate ions can move to the material in the aqueous processes (e.g., during glazing by immersion) due to diffusive and convective transport. These ions may crystallise at the surface or inside the material when environmental conditions change, such as temperature [5], and they may cause severe and repetitive damage to porous materials due to crystallisation and hydration pressure, and also thermal expansion. Individual or the combined effects of these factors generate interior stresses within the material, eventually leading to microscopic defects [6-9]. Moreover, the ion release of soluble borates in aqueous suspensions negatively affects the dispersion stability [10]. Besides, when the borates react with water and transform into their hydrate forms, they dissolve faster than their anhydrous forms. Additionally, soluble borates can absorb water from the atmosphere during storage due to their hygroscopic nature, resulting in the possibility of inaccurate amounts being incorporated into a formulation [11]. In summary, moisture absorption, water-solubility, and hydration tendency of some borates, such as anhydrous borax, limit their utilisation in the water-based systems. Therefore, one must reveal an approach to avoid water interacting with the boron oxide particle.

The preferred powders in the manufacturing process may not have tailored properties, which will allow it to be immediately introduced into a process, or may not be a naturally occurring material. In such cases, powders may need to be functionalized [12]. The powder coating, which is one of the functionalization methods of powders, is finding widespread application in a variety of industries, including pharmaceuticals, biomedical, food, detergent, fertiliser, cosmetics, and minerals to improve their properties such as flowability, wettability and dispersibility [13], and also hydration resistance [14]. The coating process includes the covering of particulate materials such as powders, 
pellets, and agglomerates with a surrounding layer of a modifier agent. The uniform coatings in the range of a few nanometers to a few microns in thickness can be applied to a variety of substrates ranging from submicron particles to millimetres. The modifier agent can be introduced to the system in solid, liquid, or suspension forms. Generally, from this point of view, coating processes can be classified as wet coating, dry coating, and melt coating [15].

Nowadays, most commercial hybrid powders are produced via wet methods such as dip, spin, and spray coating, where the modifier agents are dissolved or dispersed in a suitable solvent. The environmental hazards of wet methods have forced researchers to find alternative ways to modify powders [16]. Dry coating techniques are becoming an attractive method because they present a facility to obtain surface properties that satisfy application requirements [17]. Contrary to wet coating techniques, dry coating processes do not require any solvents and binders to remove by drying, thus provide reduced production costs and energy savings [14].

The dry coating is based on the principle of ordered mixing, introduced by Hersey. Ordered mixing occurs because of physico-chemical surface interactions between particles of highly different sizes. This principle explains that fine and cohesive particles, when properly dispersed in the powder blend; tend to stick to the surface of coarse particles, mainly due to van der Waals interactions $[18,19]$. When the ratio of diameters is high enough, the adhesive forces associated with surface attraction become greater than the repulsive forces applied by the environment on the particles, mainly associated with gravity and inertia. The resulting powder is then composed of similarly structured units where the fines are coated on the surface of the coarser particles [19]. The dry coating is achieved through mechanical forces (mechanical impact, shearing, etc.), providing coverage of the guest particles $(0.1-50 \mu \mathrm{m})$ over the host particle $(1-500$ $\mu \mathrm{m})$ [20-22]. Coating success depends on interactions between particles of the guest and host materials, and the applied force to bring them together. A sustained or discrete coating can be produced depending on a variety of operating conditions, including activation time, mechanical action, the weight fraction of guest to host particles, and the physical properties of the particles used $[13,15]$. The coating durability varies considerably depending on powders' properties and mainly the coating process used. Several systems have been developed for dry coating such as Mechanofusion, Hybridizer, and Cyclomix for applying a high level of mechanical forces, or Theta Composer and V-Blender for applying a small level of mechanical forces $[14,17,19]$. The coating systems where high levels of mechanical forces are applied [16], such as hybridizers and mechanofusion, generate local temperature rise [14] compared to other systems. This type of devices is making the coating processes unfavourable for unstable modifier agents, which degrade when exposed to such conditions.

As an alternative tool for dry coating, a laboratoryscale planetary milling device was used by Sonoda et al. [23]. This device operates in the centrifugal mode, which leads to a more gentle size reduction process with fewer abrasions. Besides, the difference in speeds between the balls and the grinding medium produces high-impact forces. These forces provide the possibility of very efficient mixing and coating in the planetary ball mill [24-26].

Some organic surface-active agents such as fatty acids and their derivatives, silane coupling agents, titanate coupling agents, and some organometallic compounds can be used for dry or wet coating of inorganic surfaces [27]. A variety of inorganic powders (such as calcite [28-30] and magnesium hydroxide [28,31], etc.) were used in the dry coating studies with stearic acid. Stearic acid $\left(\mathrm{C}_{17} \mathrm{H}_{35} \mathrm{CO}_{2} \mathrm{H}\right)$ is the highest molecular weight saturated fatty acid with the melting point of $69.6^{\circ} \mathrm{C}$ [32], and containing 18 carbons [33]. This long carbon chain ( $>12$ carbons) makes it insoluble in water [33]. Stearic acid is a low-cost organic acid, and it has been used as the mineral modifier [34]. It is generally considered that the interaction between mineral and stearic acid is physical adsorption [35].

The industrial applications of some borates are limited due to their solubility behaviour in aqueous systems and cause some application failures unless the preventive precautions against interaction with water are taken. The high-cost fritting process is the only industrial method currently applied to make water-soluble borates insoluble. Despite its commercial importance, an industrially alternative method to the fritting process to prevent borates' solubility has not yet been found. However, a few studies on the production of lower-cost calcined borate, as an alternative approach, are available in the literature [10].

Although dry coating techniques have been identified as appropriate for the coating of many oxides surfaces, there has been no study in the literature on the surface coating of borates until recently. On the other hand, very recently, in our previous study on the dry coating of anhydrous borax powders in the planetary ball mill [36], it has been found that promising results can be achieved by the dry coating of ANB powders with magnesium stearate, as an approach to overcome the above-mentioned restrictive conditions. However, to reduce the solubility of anhydrous borax in water, there is a still need in the dry coating process for improvement by adjusting the process parameters such as the modifier agent and its amount. 
This study's objective is to develop a simple, potentially cost-effective technique for the coating hydrophobic entities onto the surface of anhydrous borax particles to reduce the solubility of borates in aqueous systems. The main idea is that the coating with hydrophobic materials will delay the transformation of borates into hydrate forms, hence decreasing solubility and increasing the durability in aqueous systems. Stearic acid, which is cheaper and purer fatty acid than magnesium stearate as the modifier agent, and various process parameters for the dry coating system, were used to accomplish this goal. The processed powders were characterized by their morphology and physical, chemical, and thermal properties. The performances of the raw and processed powders in the aqueous system were also compared.

\section{Materials and methods}

\subsection{Materials}

Anhydrous borax (ANB) powders $\left(\mathrm{Na}_{2} \mathrm{~B}_{4} \mathrm{O}_{7}\right)$ of purity $99 \%$ and particle size less than $500 \mu \mathrm{m}$ was provided by Eti Mine Enterprises (Turkey). ANB powders were dry-coated with $0.5,1$, and 2 wt $\%$ stearic acid (SA) powders to create a protective layer on their surfaces in the planetary ball mill. Stearic acid (SA), encoded as Wilfarin SA-1865, was provided in the form of powder with a particle size $<10 \mu \mathrm{m}$, and the average particle size was 2 microns. The main properties of the stearic acid powders are presented in Table 1.

Table 1. Technical data of stearic acid.

\begin{tabular}{lc}
\hline Property & Value \\
\hline Formula & $\mathrm{CH}_{3}\left(\mathrm{CH}_{2}\right)_{16} \mathrm{COOH}$ \\
Molecular weight $(\mathrm{g} / \mathrm{mol})$ & 284.47 \\
Boiling point $\left({ }^{\circ} \mathrm{C}\right)$ & 383 \\
Melting point $\left({ }^{\circ} \mathrm{C}\right)$ & 69.08 \\
Density $\left(\mathrm{gr} / \mathrm{cm}^{3}\right)$ & 0.941 \\
Solubility in water $(\mathrm{g} / 100 \mathrm{~mL})$ at $25^{\circ} \mathrm{C}$ & 0.034 \\
\hline
\end{tabular}

\subsection{Dry coating processes}

As a practical and effective way to surface modification, a dry process was preferred for coating powders by using a planetary ball mill, which consists of a ceramic jar (volume $500 \mathrm{~mL}$ ) filled with alumina balls (diameter $10-20 \mathrm{~mm}$ ). The rotational speed of the jar is $350 \mathrm{rpm}$, and a ball/powder weight ratio of 2:1 is used. The jar also contains a free volume of approximately $60 \%$ for the movement of powders. The dry coating procedure of stearic acid onto anhydrous borax powder's surface in the planetary ball mill was summarised as follows.

Prior to the coating process, each $100 \mathrm{~g}$ of ANB was mixed manually with three different amounts of $S A(0.5$, 1 , and $2 \mathrm{wt} \%)$. For each coating experiment, the powder mixtures were fed into the milling jars and treated at 30,60 , and $120 \mathrm{~min}$ in the working chamber of the laboratory-scale milling device. After being processed for the prescribed application mode, the powders were kept for further measurements and characterisations.

\subsection{Powder characterisation}

In order to investigate the effects of the dry coating process on the properties of anhydrous borax, the particle size, shape, and surface morphology of surfacecoated and uncoated ANB powders were characterised by particle size distribution and scanning electron microscopy. The solubility tests to determine the coating efficiency of stearic acid on anhydrous borax powders, as well as the wettability and dispersibility tests to examine the effects of that coating on the properties of ANB powders, were performed. Afterwards, the powders having high coating efficiency were subjected to further characterisation using different analytical techniques such as X-ray diffraction and photoelectron spectroscopy, Fourier-transform infrared spectroscopy, and Differential thermal and thermogravimetric analysis.

\subsubsection{Measurement of the particle size distribution}

The particle comminution would be the most important factor in determining the optimum coating conditions. Therefore, the size distributions of anhydrous borax powders were determined using a laser particle size analyser (Mastersizer 2000, Malvern Instruments Ltd., Worcestershire, UK).

The particle size distribution of SA-coated and uncoated ANB powders was measured and compared to understand the impact of the dry coating process. Three measurements were performed for each experiment, and the mean and standard deviation were calculated.

\subsubsection{Scanning electron microscopy analysis}

The shape and surface morphology of ANB powders, before and after dry coating, was observed through scanning electron microscopy (SEM, Leo 1430 VP). During the observation, secondary electron images (SEI) at different magnifications of ANB powders were obtained. Energy-dispersive X-ray spectroscopy (EDX) analysis was also conducted to prove the SA coating presence on ANB surfaces.

\subsubsection{Measurement of water-ANB particle contact angles}

Evaluation of the effectiveness of stearic acid coating on reducing water interaction of ANB was firstly performed by wettability tests. The wettability of the powder samples was measured at $25{ }^{\circ} \mathrm{C}$ with the sessile drop method using a digital goniometer (KSV Attension ThetaLite TL 101). The sessile drop method, which is based on measuring the contact angle by depositing a small drop of pure water $(\sim 5 \mu L)$ on samples, was 
used to determine the wettability of anhydrous borax powders. Three measurements were conducted at different locations on each sample, and the average values of the measured contact angles were noted.

\subsubsection{Determination of the coating efficiency}

Coating efficiency evaluation involves solubility tests, in which the powders were first dispersed into $100 \mathrm{~mL}$ of distilled water, and vigorously mixed by a magnetic stirrer at $750 \mathrm{rpm}$ for $60 \mathrm{~min}$ at room temperature. The solutions were filtered through a white ribbon filter paper, and accumulated powders on the filter paper were dried in an oven at $50{ }^{\circ} \mathrm{C}$ until the weight became constant. Subsequently, dried powders were gently scraped off the filter paper with a spatula. The amounts of maximum soluble powders $\left(m_{0}\right)$, insoluble parts of surface-coated $\left(m_{1}\right)$ and uncoated ANB powders $\left(m_{2}\right)$ were weighed accurately for each set of parameters, and coating efficiency (\%) was determined using the following equation (Eq.1).

$$
\text { Coating efficiency }(\%)=\left[\left(\mathrm{m}_{1}-\mathrm{m}_{2}\right) / \mathrm{m}_{0}\right] \times 100
$$

\subsubsection{Fourier-transform infrared and X-ray photo- electron spectroscopies analysis}

In order to detect possible structural changes after the dry coating process, Fourier-transform infrared (FT-IR) and X-ray photoelectron spectroscopies (XPS) were used. Infrared spectra of the surface-coated and uncoated ANB powders were observed using the PerkinElmer Spectrum BX spectrophotometer with a resolution of about $4 \mathrm{~cm}^{-1}$ from 4000 to $500 \mathrm{~cm}^{-1}$. An X-ray photoelectron spectrometer (XPS, Thermo Scientific, USA) with a monochromatic Al-Ka X-ray source $(1486.7 \mathrm{eV})$ having a spot size of approximately 400 $\mu \mathrm{m}$ was also used for the spectral measurements. Survey spectra, from -10 to $1350 \mathrm{eV}$ binding energy, were recorded at $150 \mathrm{eV}$ pass energy with an energy step of $1.0 \mathrm{eV}$ with a typical average of 20 scans.

\subsubsection{Differential thermal and thermogravimetric analysis}

The dry coating mechanism was also evaluated based on the thermal stability of ANB powders before and after dry coating using the simultaneous differential thermal analyser (DTA, Netzsch STA 449 F3 Jupiter, Germany). For thermal analyses, the powders were heated in the air atmosphere at a rate of $10{ }^{\circ} \mathrm{C} / \mathrm{min}$ from 25 to $1000{ }^{\circ} \mathrm{C}$. The thermogravimetric analysis (TGA) profile and measurement of the variation in weight loss with respect of temperature were obtained by the software automatically.

\subsubsection{X-ray diffraction analysis}

The impact of SA coating on the preventing hydration of ANB was investigated by X-ray diffraction (XRD) analysis. Prior to analysis, surface-coated and uncoated
ANB powders were subjected to the washing treatment, and afterward, the structural changes owing to likely hydration were evaluated using an X-ray diffractometer with Cu-Ka radiation (XRD, Bruker-D8 Advance, Germany). The diffractograms were obtained in the range of $2 \theta=10^{\circ}-80^{\circ}$ with a scan rate of $3^{\circ} / \mathrm{min}$.

\subsubsection{Rheological measurements}

The effect of stearic acid coating onto the surface of anhydrous borax on its dispersibility in the waterbased suspension was evaluated by rheological measurements using a stress-controlled rheometer (Anton Paar, MCR 102, Germany). All the rheological measurements were performed as a function of shear rate (1-1000 s-1), at $25^{\circ} \mathrm{C}$ using the parallel plate geometry with $50 \mathrm{~mm}$ diameter plates. Two kinds of aqueous dispersants (Darvan 821A and Dolapix CE64) were used to stabilise the glaze suspensions containing surface-coated and uncoated ANB powders. Rheological measurements were conducted to determine the dispersibility of ANB powders within the as-prepared suspensions using three different concentrations of each dispersant $(0.1,0.3$ and $0.5 \mathrm{wt} \%)$. All the glaze constituents were premixed together with water (35 wt \%) by a magnetic stirrer at $750 \mathrm{rpm}$ for $15 \mathrm{~min}$ in order to prepare the suspensions. Afterward, the dispersants at the stated concentrations were added to the premixed suspension. Finally, surface-coated and uncoated ANB (10 wt \%) powders were added to the suspensions under stirring, and the mixture was stirred at a speed of $1200 \mathrm{rpm}$ for a further $15 \mathrm{~min}$.

\section{Results}

\subsection{The impact of the coating period on the par- ticle size and solubility of ANB powders}

ANB powders were processed with the planetary ball mill at the previously defined periods, and the corresponding $d_{10}, d_{50}$ and $d_{90}$ values, which indicate the size of the fine $\left(d_{10}\right)$ and coarse $\left(d_{90}\right)$ fractions, and of the median particle size $\left(d_{50}\right)$, for each amount are summarised in Table 2. The $d_{90}$ value of as-received ANB powders was determined to be less than $500 \mu \mathrm{m}$. The impact of the used mechanical force on the size of uncoated ANB particles was proved by a considerable reduction in the $d_{90}$ value $(<100 \mu \mathrm{m})$ after a processing period of $120 \mathrm{~min}$. On the other hand, the $d_{10}, d_{50}$, and $d_{90}$ values of SA-coated powders decreased significantly compared with that of the uncoated powders. This finding seems similar to the results reported in our recent work [36]. In our previous study, the $d_{90}$ value for anhydrous borax particles, which were coated with 1 wt $\%$ MgSt (magnesium stearate) for $120 \mathrm{~min}$, was determined to be reduced by $16 \mu \mathrm{m}$ compared with that of the uncoated particles under the given conditions. However, in contrast with the results reported in that work, it was found here that $d_{50}$ and $d_{10}$ values were also decreased. Koskela [37] claims that there may be 
a decrease in the $d_{90}$ values, which indicate the host particles may have split into smaller pieces during the coating process. Moreover, it was also claimed that the small particle sizes of the host particles less sensitive to the high shear forces of the coating process. The observed reduction in the particle sizes of surfacecoated ANB can also be explained by the presence of stearic acid, which helps decrease friction between the balls and the wall of the grinding jar [38].

As seen in Table 2, there was no significant change in the particle size of SA-coated ANB powders with an increase in the amount of SA for the processing time of 30 minutes. With the increase in the amount of SA, on the other hand, it appears that the $d_{90}$ values for the processing time of 60 minutes, and $d_{90}$ and $d_{50}$ values for the processing time of 120 minutes increased. However, when the amount of SA is used as $2 \mathrm{wt} \%$ and processing period was set to 60 and $120 \mathrm{~min}$, it is seen that large and medium-sized ANB powders cannot be further split into a smaller size. As a result, it can be said that the particle size of the coated ANB is reduced by the lubricating effect of $S A$, which reduces friction in the coating medium, and by the exposure of the powders to excessive impact movements with increasing process time.

The particle size of ANB powders also significantly affects its water solubility, as seen in Figure 1. The weight loss of raw ANB in water, an indicator of solubility, was measured to be $27.3 \%$. Although there was no remarkable difference in weight loss up to processing time of 60 minutes, it reached the level of $40 \%$ after treatment for $120 \mathrm{~min}$. Contrary to processed ANB powders not coated with SA, weight loss decreased to $8.6 \%$ in $1 \mathrm{wt} \%$ SA-coated ANB for $60 \mathrm{~min}$. These finding points out that SA particle were, to a certain extent, bonded to the surface of ANB powders and relatively prevented its solubility.

When this result is compared with the results obtained in our study on the dry coating of anhydrous borax with

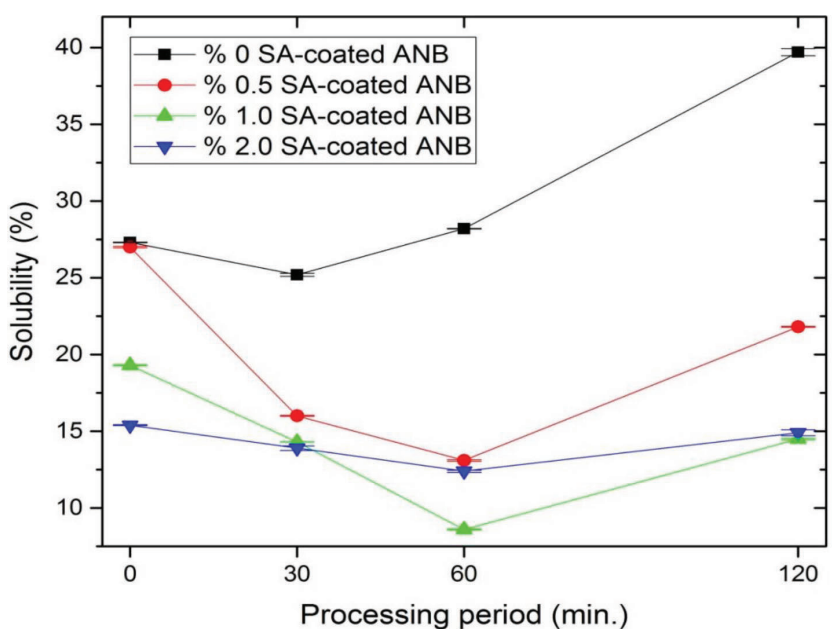

Figure 1. Effect of processing parameters on the solubility of ANB powders.

Mg-St [36], weight loss due to solubility in water after $120 \mathrm{~min}$ processing time with $1 \mathrm{wt} \% \mathrm{Mg}$-St was measured as $10.5 \%$. In this study, a lower weight loss value $(8.6 \%)$ was obtained by coating the same amount of stearic acid in shorter processing time (60 min). As a result, it was found that stearic acid gives better results in the dry coating of anhydrous borax than Mg-St.

\subsection{The effect of dry coating on the surface mor- phologies of ANB powders (SEM analysis)}

Scanning electron microscopy (SEM) through image analysis was done to confirm the SA coating presence on ANB surfaces. SEM images of uncoated powders indicate that ANB has angular and mostly not aggregated particles (Figure 2a, 2c). SEM images of the coated ANB powders with $1 \mathrm{wt} \% \mathrm{SA}$ for $60 \mathrm{~min}$ are given in Figure $2 b, 2 d$, and $2 e$. During the observations, no agglomerated free stearic acid particles were observed on the support, and it was also observed that the particles keep their structure morphologies instead of becoming completely spherical. On the other hand,

Table 2. Particle sizes of ANB powders as a function of processing parameters.

\begin{tabular}{ccccc}
\hline \multirow{2}{*}{$\begin{array}{c}\text { SA } \\
\text { amount } \\
\text { (wt. } \%)\end{array}$} & $\begin{array}{c}\text { Processing } \\
\text { period } \\
(\mathbf{m i n})\end{array}$ & \multicolumn{3}{c}{ Particle size $(\boldsymbol{\mu m})$} \\
\cline { 3 - 5 } & 0 & $\mathbf{d}_{\mathbf{1 0}}$ & $\mathbf{d}_{\mathbf{5 0}}$ & $\mathbf{d}_{\mathbf{9} 0}$ \\
\hline 0 & 30 & $20.8 \pm 0.77$ & $267.6 \pm 0.81$ & $499.3 \pm 0.33$ \\
\hline 0 & 30 & $10.4 \pm 0.32$ & $110.0 \pm 0.84$ & $328.9 \pm 0.49$ \\
0.5 & 30 & $11.6 \pm 0.69$ & $88.4 \pm 0.52$ & $220.4 \pm 0.21$ \\
1.0 & 30 & $13.6 \pm 0.65$ & $90.0 \pm 1.00$ & $238.3 \pm 2.34$ \\
2.0 & 60 & $12.4 \pm 0.35$ & $72.3 \pm 0.19$ & $178.6 \pm 0.15$ \\
\hline 0 & 60 & $3.7 \pm 0.03$ & $47.9 \pm 0.15$ & $108.0 \pm 0.35$ \\
0.5 & 60 & $3.1 \pm 0.75$ & $66.8 \pm 1.90$ & $165.9 \pm 1.80$ \\
1.0 & 60 & $2.8 \pm 0.03$ & $67.2 \pm 1.53$ & $183.5 \pm 1.45$ \\
2.0 & 120 & $6.5 \pm 0.29$ & $40.6 \pm 0.62$ & $100.6 \pm 0.42$ \\
\hline 0 & 120 & $2.3 \pm 0.10$ & $19.9 \pm 0.68$ & $51.6 \pm 0.40$ \\
0.5 & 120 & $2.2 \pm 0.05$ & $30.1 \pm 0.22$ & $78.2 \pm 0.63$ \\
1.0 & 120 & $1.4 \pm 0.18$ & $36.5 \pm 1.53$ & $105.7 \pm 1.75$ \\
2.0 & & & &
\end{tabular}


SEM images at low magnifications did not allow the particles of ANB and SA being well-discriminated from each other. However, the discrete coating of SA on the ANB surface was observed at a magnification over $5000 X$ with the aid of EDX analysis, as represented in Fig. $2 \mathrm{f}$, confirmed the existence of the SA coating on the ANB surfaces.
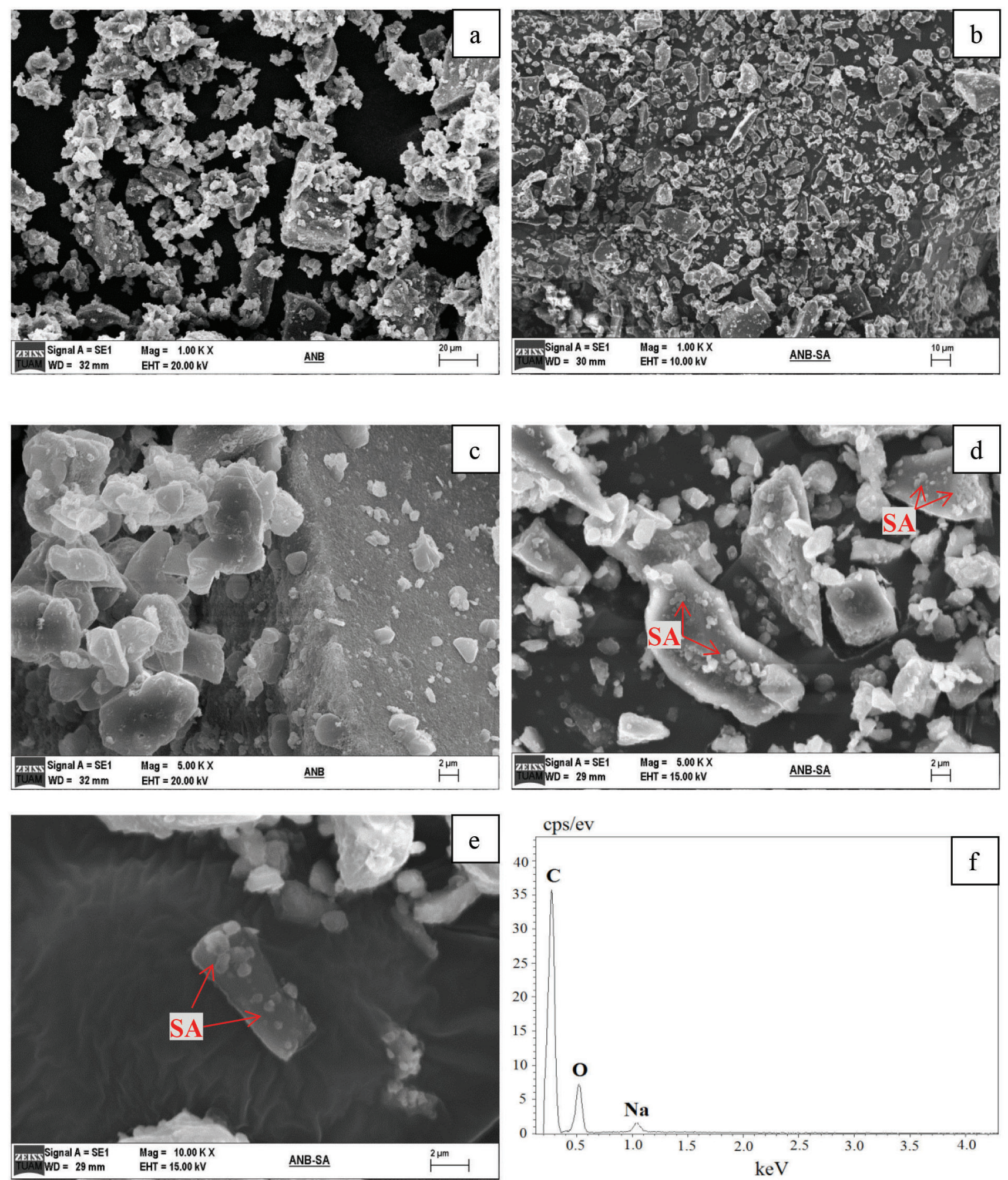

Figure 2. Scanning electron micrographs of ANB powders at different magnifications; uncoated ANB powders at a magnification of $1000 \mathrm{X}$ (a) and $5000 \mathrm{X}$ (c), ANB powders coated with $1 \mathrm{wt} \%$ SA for $60 \mathrm{~min}$ at a magnification of 1000X (b), 5000X (d) and 10000X (e), EDX analysis of ANB powders coated with $1 \mathrm{wt} \%$ SA for $60 \mathrm{~min}(\mathrm{f})$. 
the range $17-99.28^{\circ}$, and the highest value was measured for the samples surface-coated ANB with $1 \mathrm{wt} . \%$ SA for $60 \mathrm{~min}$. High measured values of contact angles for all the surface-coated samples revealed significant hydrophobicity compared with that of uncoated ANB. When the contact angle results are compared with the results obtained in the solubility test, the dry-coated ANB powders with 1 wt $\%$ SA for 60 min provided the best results in both test results.

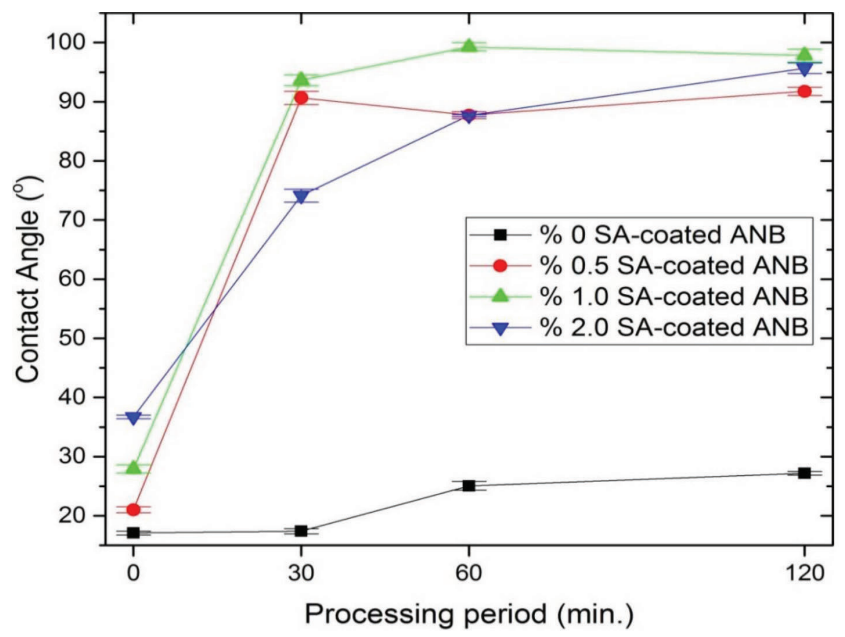

Figure 3. Contact angles of surface-coated and uncoated ANB powders.

\subsection{Coating efficiency of stearic acid on anhy- drous borax}

As an approach to the evaluation of coating efficiency, the insolubility values of surface-coated and uncoated ANB powders were determined through the watersolubility experiment. The uncoated ANB was partially water-soluble, and the amount of its insoluble fractions was $73 \%$. When the surface of ANB was coated with SA, the amount of insoluble fractions for all the samples was above $78 \%$ (Figure 4). The experimental findings indicate that stearic acid particles were partially bonded to ANB powder surfaces. The maximal insolubility of $91 \%$ was gained for ANB coated with 1 wt $\%$ SA for $60 \mathrm{~min}$.

Coating efficiency values calculated using Eq. (1) is given in Figure 5. It is seen that the results of coating efficiency vary significantly when each of the process parameters is changed. The coating efficiency varies between $36.5 \%$ and $68.1 \%$, and the maximum coating efficiency was achieved for the sample coated with $1 \mathrm{wt} \% \mathrm{SA}$ for $60 \mathrm{~min}$. In the coating processes performed with 0.5 and $1 \mathrm{wt} \% \mathrm{SA}$, the coating efficiency increased by up to processing period of $60 \mathrm{~min}$, and then decreased again for $120 \mathrm{~min}$. However, when the amount of SA is used as 2 wt $\%$, the coating efficiency increased depending on the increase of the coating period. When the two extreme times (60 and $120 \mathrm{~min}$ ) are compared in terms of coating efficiency, the ratio of guests to the host particle seems to be optimum

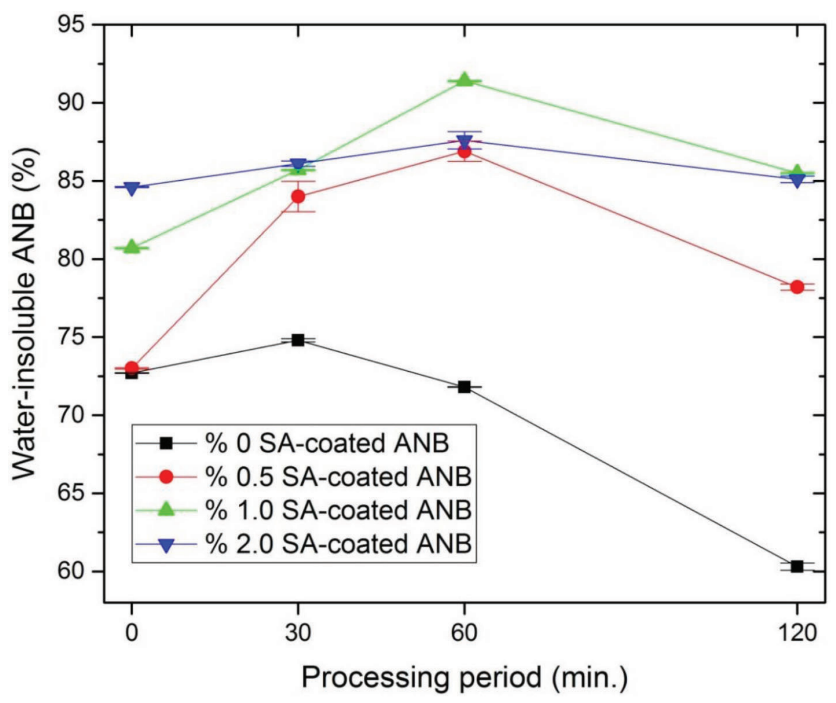

Figure 4. Water-insoluble ANB powders amount (\%) as a function of the processing period.

for a processing time of $60 \mathrm{~min}$; whereas, the initial stearic acid amount in the two samples is the same. Two phenomena could explain these results: first, the agglomeration of the stearic acid particles and second, decrease of the adhesion force of SA particles on the ANB particles.

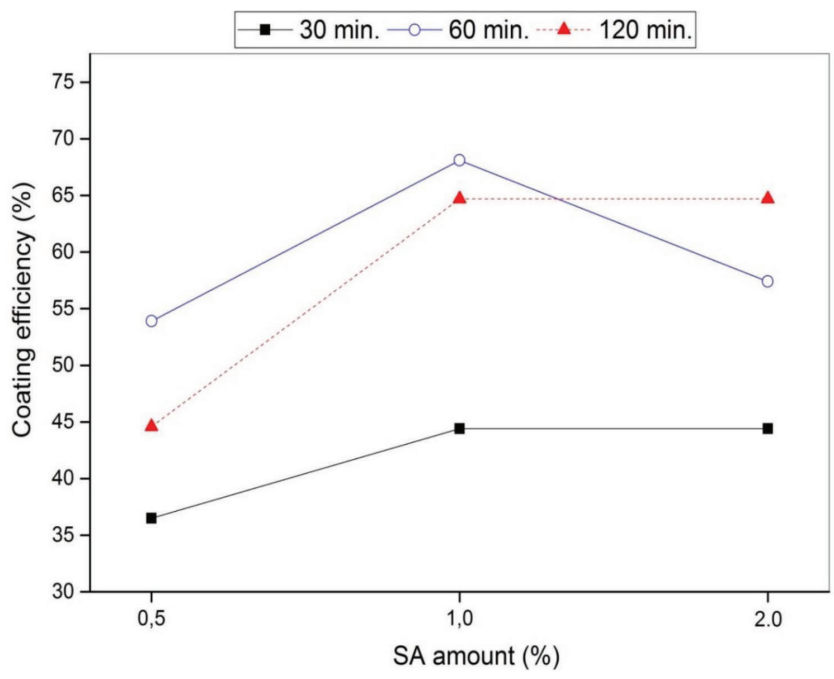

Figure 5. Effect of processing parameters on coating efficiency of SA.

\subsection{FT-IR and XPS analysis of surface-coated and uncoated ANB powders}

FT-IR spectroscopy is a practical analysis instrument to examine the interaction between active groups at the molecular level. The FT-IR spectra of surface-coated and uncoated ANB powders are shown in Figure 6 . The IR spectra of both samples exhibit the same bands in the range $500-1600 \mathrm{~cm}^{-1}$, which are the characteristic vibrations of borax. The weak band at 709 $\mathrm{cm}^{-1}$ indicates the B-O-B ring bending. The bands at $825,997,1076$, and $1132 \mathrm{~cm}^{-1}$ are assigned to the stretching of tetragonal $\left(\mathrm{BO}_{4}\right)$ units. The other bands at $945,1283,1337$, and $1426 \mathrm{~cm}^{-1}$ are attributed to the stretching of trigonal boron $\left(\mathrm{BO}_{3}\right)$ groups. On the other 
hand, the stretching vibration was observed at 3355 $\mathrm{cm}^{-1}$, which ascribed to $\mathrm{OH}$ bonds, likely owing to the absorption of moisture from the atmosphere during storage. These assignments are consistent with the related literature $[39,40]$.

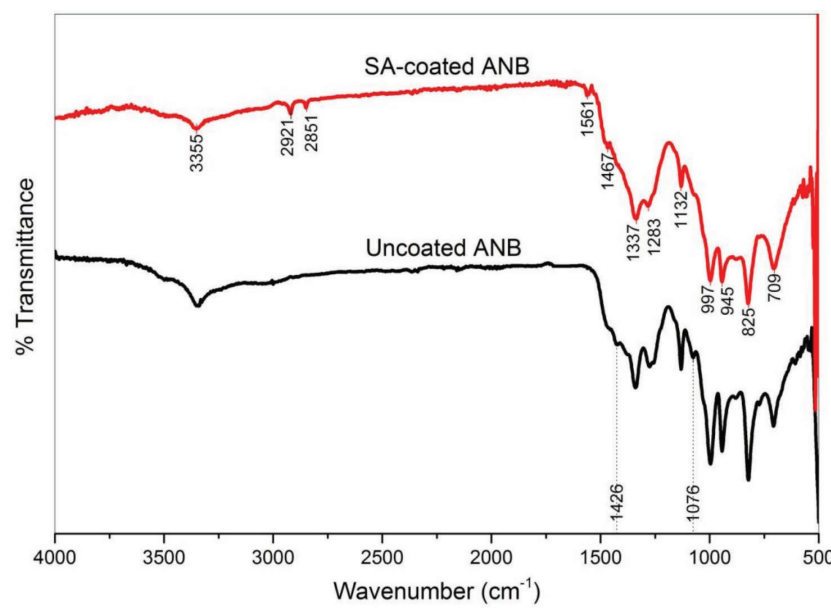

Figure 6. FT-IR spectra of surface-coated and uncoated ANB powders.

In the high-frequency region, asymmetric and symmetric stretching vibration of aliphatic groups $\left(-\mathrm{CH}_{2}-\right.$ groups) appears clearly at 2851 and $2921 \mathrm{~cm}^{-1}$ in the spectra of SA-coated ANB, which confirms the presence of stearic acid on the surface of ANB. This observation is consistent with the result obtained by Focke et al. [41]. In the low-frequency region, the bands at 1467 and $1561 \mathrm{~cm}^{-1}$ are assigned to -COO- stretching vibrations in $\mathrm{CH}_{3}\left(\mathrm{CH}_{2}\right)_{16} \mathrm{COO}$ - groups, implying that the stearic acid on the surface of ANB. This finding is also consistent with the result obtained by Qing et al. [42].

When comparing the FT-IR spectra of samples, a slight decrease in the intensities of characteristic bands of borax was observed. Additionally, the weak bands at 1076 and $1426 \mathrm{~cm}^{-1}$ were disappeared after surface modification. No additional and shifted bands were also observed in the spectra of SA-coated ANB, and this indicates that there is no chemical interaction between SA and ANB, thus proving the inertness of ANB, which only behaves as a host carrier. As a result, it was concluded that the coating of stearic acid to the surface of anhydrous borax is a physical bonding rather than chemical bonding.

The elemental surface compositions, as probed by XPS analysis, confirming the existence of SA on ANB, are presented in Figure 7 and Table 3 . After treating the powder surfaces with SA via the dry coating, the presence of carbon element, which is the primary indicator of stearic acid in the investigation of the surface chemistry of ANB samples, was evaluated. XPS wide-scan spectra of the raw and surface-coated ANB powders indicate the spectrums are dominated by $\mathrm{B}$, $\mathrm{Na}$, and $\mathrm{O}$ signals, as expected since borax is formed from these elements. A strong $\mathrm{C}$ 1s peak was detected in SA-coated ANB powders, while a weak C1s signal, owing to likely due to carbonaceous contamination, and were observed in the raw ANB powders.

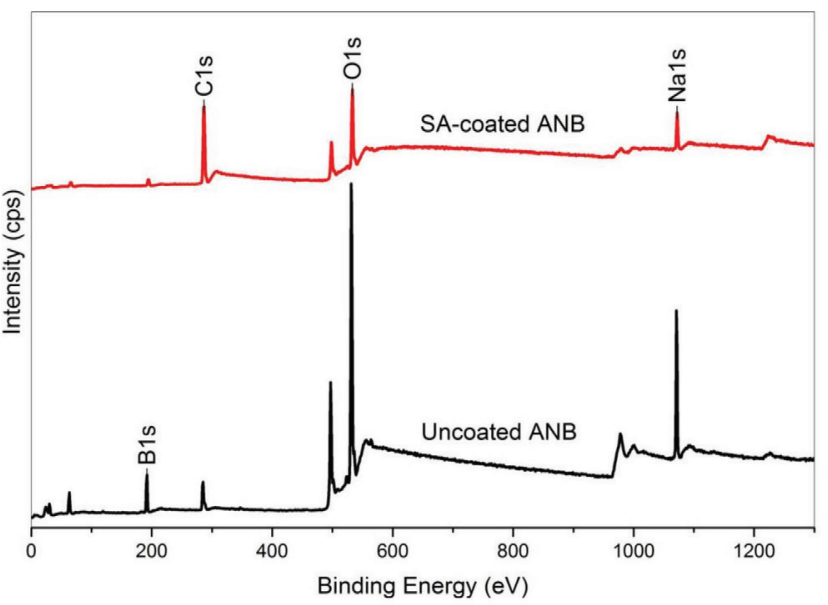

Figure 7. XPS analysis of surface-coated and uncoated ANB powders.

Table 3. Surface composition of surface-coated and uncoated ANB powders.

\begin{tabular}{lcccc}
\hline \multirow{2}{*}{ Sample } & \multicolumn{4}{c}{ Atomic percent of elements (at. \%) } \\
\cline { 2 - 5 } & $\mathrm{B} 1 \mathrm{~s}$ & $\mathrm{O} 1 \mathrm{~s}$ & $\mathrm{Na1s}$ & $\mathrm{C} 1 \mathrm{~s}$ \\
\hline Raw ANB & 25.19 & 47.90 & 11.05 & 12.34 \\
SA-coated ANB* & 8.01 & 26.53 & 9.44 & 56.02 \\
\hline
\end{tabular}

*: 1 wt. \% SA-coated ANB for $60 \mathrm{~min}$.

\subsection{Thermal stabilities of surface-coated and un- coated ANB powders}

The DTA curve of uncoated ANB powders shows one exothermic peak at $540^{\circ} \mathrm{C}$ and two endothermic peaks at $150^{\circ} \mathrm{C}$ and $740{ }^{\circ} \mathrm{C}$ (Figure 8a). The endothermic peaks are attributed to the dehydration of physically adsorbed water and the fusion of crystal borax. The exothermic peak is related to the crystallisation of amorphous anhydrous borax. The amorphous form of anhydrous borax crystallises at a temperature of 575 ${ }^{\circ} \mathrm{C}$, forming a compound of the same composition. Typically, this is the process of the internal rearrangement of an amorphous structure occurring below its melting temperature. This agrees with the result obtained by Waclawska et al. [43].

At the comparatively displayed DTA curves of samples, both surface-coated and uncoated ANB followed a similar behaviour, as seen in the thermogram. However, there is a broad but slight hump-shaped curve, appeared at temperatures range between $300-500$ ${ }^{\circ} \mathrm{C}$, and this can speculate to be associated with the degradation of stearic acid. The boiling temperature of stearic acid $\left(383^{\circ} \mathrm{C}\right)$, corresponds to the maximum point of the mentioned hump-shaped curve. These observations are comparable with those of Heller-Kallai et al. [44]. 

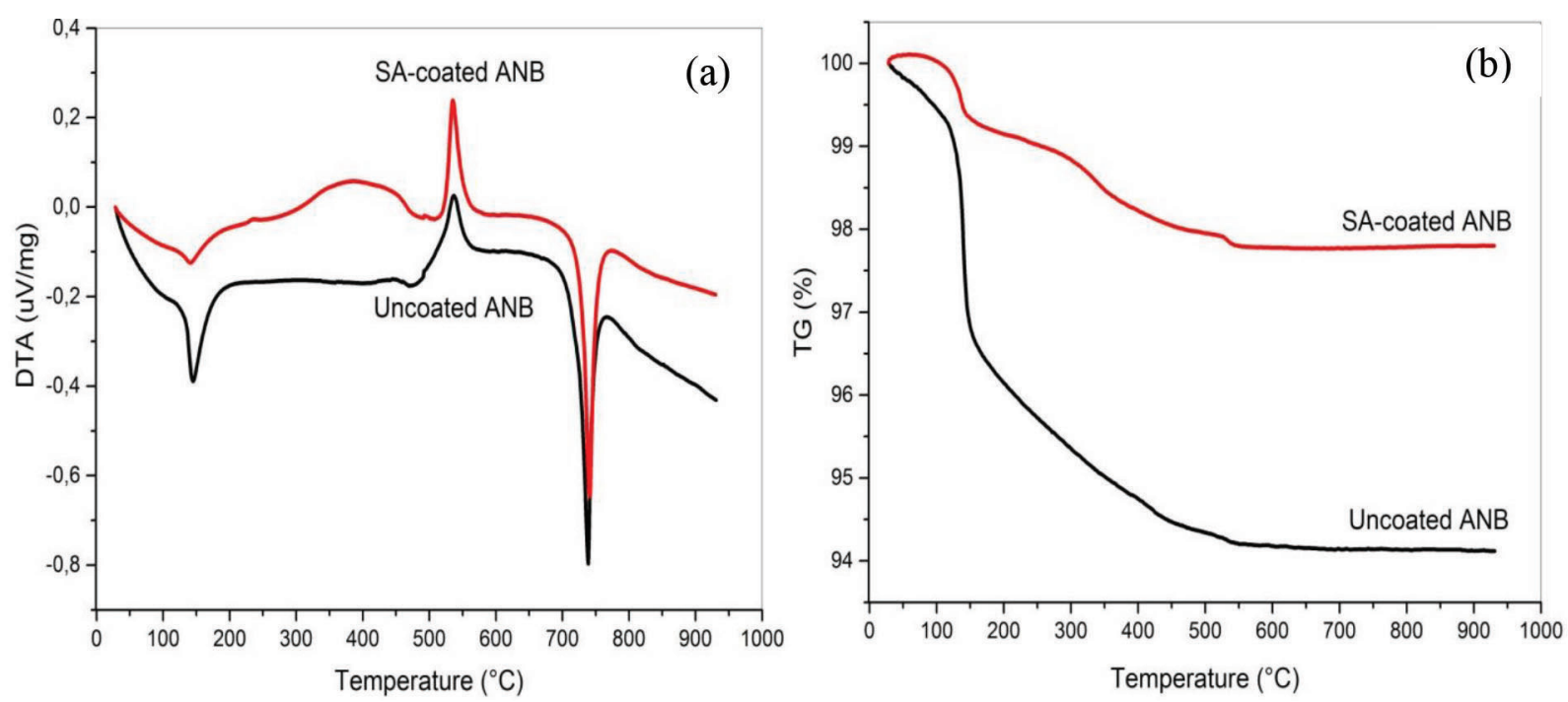

Figure 8. DTA-TG analysis of surface-coated and uncoated ANB powders; a) DTA, b) TG

TGA curves of ANB powders before and after dry coating are plotted in Figure 8b. The weight loss difference between the surface-coated and uncoated ANB powders is more noticeable. Up to $550{ }^{\circ} \mathrm{C}$, the $\mathrm{OH}$ groups are gradually removed from surface-coated and uncoated ANB powders, which are accompanied by weight loss of 5.75 and $2.0 \%$, respectively. This difference is thought to be due to the increase in weight due to the oxidation of SA during its degradation process. Furthermore, SA reduces $\mathrm{OH}$ removing, so; only physical changes in the characteristic thermal behaviour are achieved.

The results suggest that stearic acid was bound to the surface of the anhydrous borax by a physical mechanism rather than chemical bonding, due to the fact that stearic SA coating caused only a change in mass.

\subsection{Effect of dry coating on the hydration resis- tance of ANB powders}

The effect of stearic acid coating on the preventing hydration of ANB powders is noticeable, as seen in Figure 9. After washing treatment, a complete transformation into 10 moles of the water-containing form (tincal; powder diffraction file (pdf) numbers of 01-0751078) was detected in the uncoated ANB sample. In the case of SA-coated ANB, it was observed that a transformation into highly 5 moles form (tincalconite; powder diffraction file (pdf) numbers of 00-007-0277) rather than 10 moles.

The phenomenon is caused by the hydrophobic nature of the SA coating that acts as a barrier delaying the hydration of ANB. This result suggests that stearic

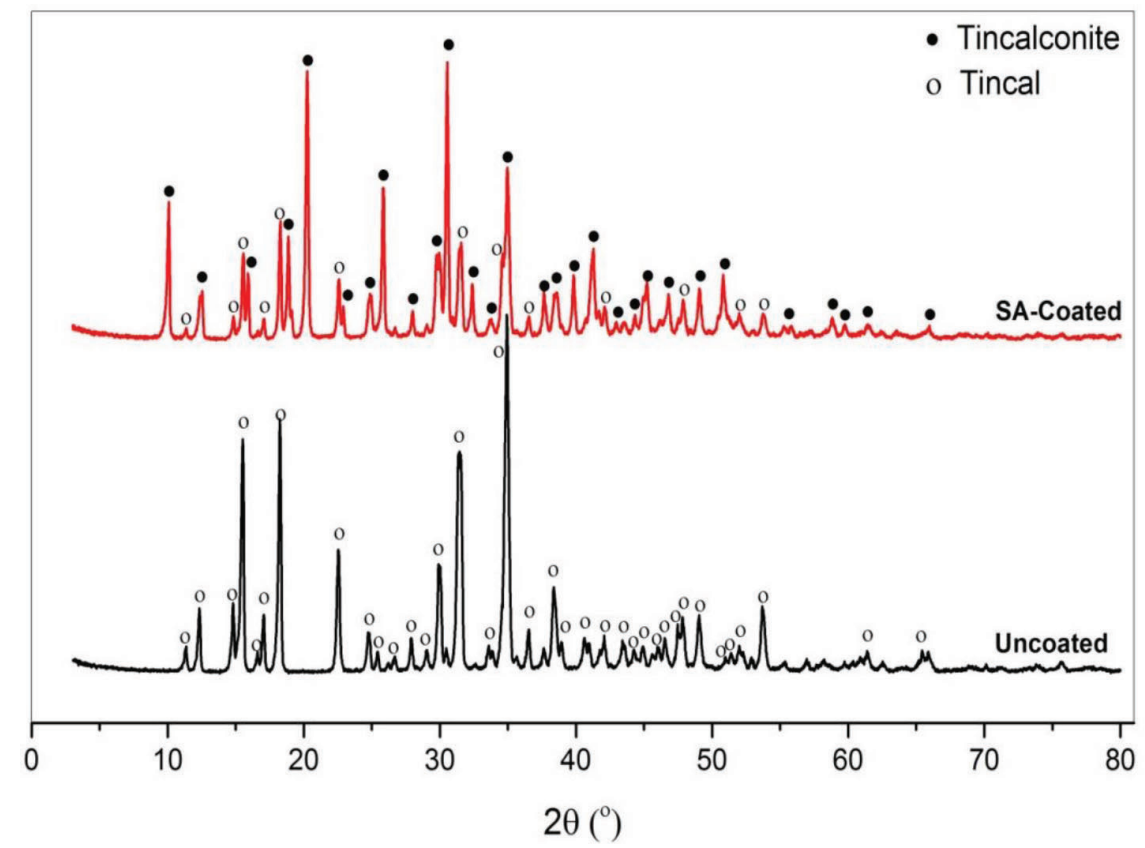

Figure 9. XRD analysis of surface-coated and uncoated ANB powders after washing treatment. 
acid was bound to the surface of the anhydrous borax because it significantly prevented further hydration. Admittedly, the expectation is to prevent the hydration completely by the produced coating. However, as observed in the SEM analysis, the discrete coating significantly reduced the hydration intensity, although it could not entirely prevent hydration.

\subsection{The stabilisation of surface-coated and uncoat- ed ANB powders in the water-based suspension}

When the flow curves of glaze suspensions containing surface-coated and uncoated ANB powders were compared, as represented Figure $10 a$ and $b$, reductions in the viscosity value and thixotropic hysteresis loop width were interpreted as the positive effect of the dry coating. Solubility and ion release of borates in aqueous suspensions negatively affect dispersion stability [11], as mentioned before. However, SA coating that acts as a barrier delaying hydration of ANB prevented further ion release by reducing dissolution. Thus, it enhanced the stability of ANB powders in suspension.

Rheological measurements also indicated that the viscosity attained the lowest value in the suspensions dispersed without Darvan 821A and Dolapix CE64. However, it has been observed that viscosity increases continually with the addition of both dispersing agents. Consequently, it has been determined that the suspensions containing ANB coated with SA have a better stabilisation compared with the suspensions containing raw $\mathrm{ANB}$, regardless of the dispersant type and amount.

\section{Discussion}

The effect of the mechanical force used in the coating system on the size of both raw and SA-coated ANB particles was quite evident. The $\mathrm{d}_{10}, \mathrm{~d}_{50}$, and $\mathrm{d}_{90}$ values of SA-coated powders decreased significantly compared with that of the uncoated form. This finding seems similar to the results reported in our recent work [36]. In our previous study on coating anhydrous borax with a dry method [36], however, the coating with magnesium stearate reduced the size of the coarse anhydrous borax grains (about $16 \mu \mathrm{m}$ compared to the uncoated form). In contrast, stearic acid, on the other hand, caused a decrease in the size of not only coarse powders but also medium and fine powders. Besides, the particle size of the coated ANB powders is being reduced by the lubricating effect of stearic acid, which reduces friction in the coating medium, and by the exposure of the powders to excessive impact forces with increasing processing time. This result is an indication that two different coating materials cause different effects in the coating environment. Unlike magnesium stearate, stearic acid has shown an impact on all particles of various sizes.

The particle size of ANB powders also significantly affects weight loss, depending on its water solubility. When the powders were processed for 60 min of processing time with $1 \mathrm{wt} \% \mathrm{SA}$, it was seen that weight loss values decreased from 40 to $8.6 \%$. As the weight loss value did not reach 0 , it was understood that SA particles were, to a certain extent, bonded to the surface of ANB powders and relatively prevented its solubility.

SEM analysis indicated that the pristine ANB powders have angular and mostly not aggregated particles. Although the mechanical forces produced in the coating system cause a decrease in the size of the particles, it has been observed that the particles retain their surface morphology and do not become being spherical. On the other hand, the discrete coating of SA on the ANB surface was observed at high magnifications, and the existence of the SA coating on the ANB surfaces confirmed with the aid of EDX analysis.
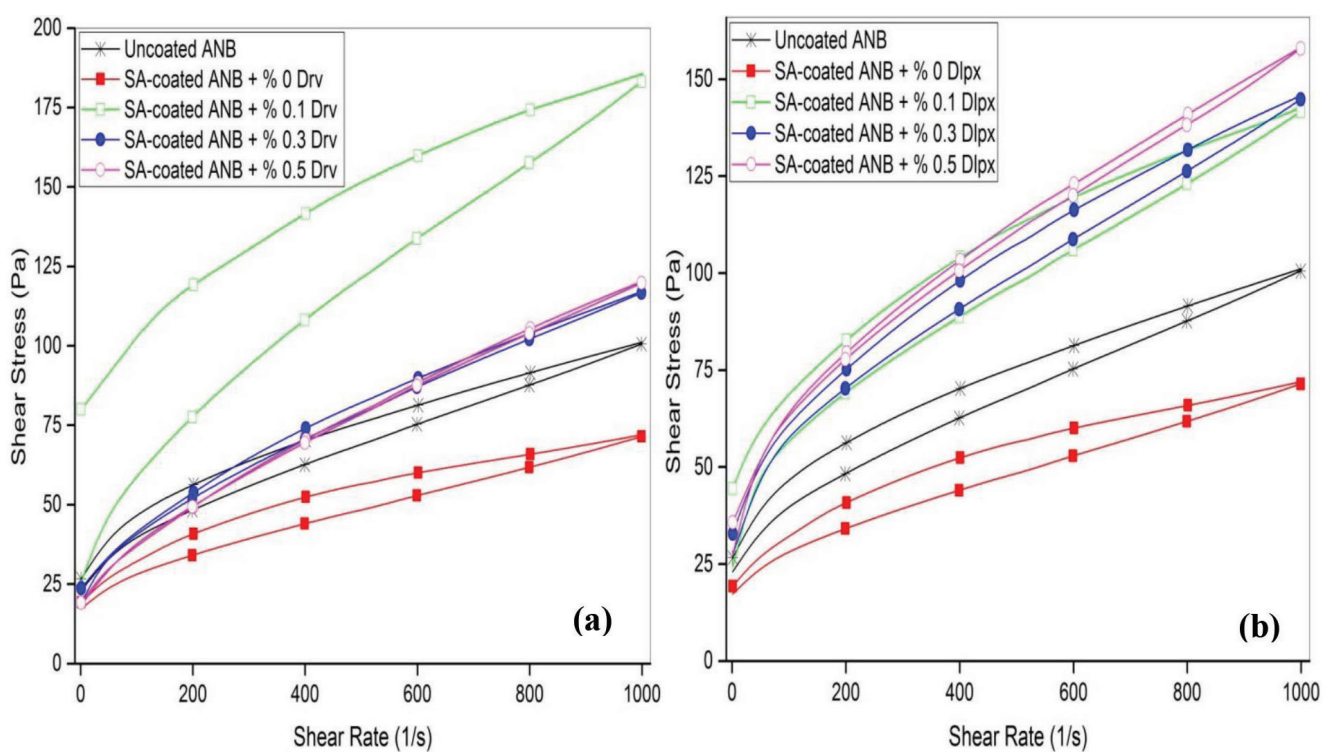

Figure 10. Flow curves of water-based suspensions containing surface-coated and uncoated ANB powders; (a) dispersed with Darvan 821A and (b) dispersed with Dolapix CE64. 
Studies of stearic acid coating on the surfaces of ANB powders by the dry method have shown that it gives substantially hydrophobic property compared to its pristine form. When the solubility and wettability properties of ANB powders were evaluated together, the dry-coated ANB powders with $1 \mathrm{wt} \%$ SA for $60 \mathrm{~min}$ were found to give the best results in both behaviors.

The coating efficiency varied significantly (from $36.5 \%$ to $68.1 \%$ ) when each of the process parameters was changed. The maximum coating efficiency was achieved for the sample coated with $1 \mathrm{wt} \%$ SA for 60 min. On the other hand, when the two extreme times (60 and $120 \mathrm{~min}$ ) are compared in terms of coating efficiency, the ratio of guest to host particle seems to be optimum for 60 min; whereas, the initial stearic acid amount in the two samples is the same. Two phenomena could explain these results: first, the agglomeration of the stearic acid particles and second, decrease of the adhesion force of SA particles on the ANB particles.

The FT-IR spectra of surface-coated and uncoated ANB powders exhibited the characteristic vibrations of borax. On the other hand, the stretching vibration of $\mathrm{OH}$ bonds $[39,40]$, likely owing to the absorption of moisture from the atmosphere during storage. The asymmetric and symmetric stretching vibration of $-\mathrm{CH}_{2}-$ groups [41] that confirms the presence of stearic acid on the surface of ANB was observed in the spectra of SA-coated ANB powders. Besides, the -COOstretching vibrations in $\mathrm{CH}_{3}\left(\mathrm{CH}_{2}\right)_{16} \mathrm{COO}-$ groups [42], implying that the stearic acid on the surface of ANB. FT-IR spectra of samples also indicated no chemical interaction between SA and ANB, thus proving the inertness of ANB, which only behaves as a host carrier. As a result, it was concluded that the coating of stearic acid to the surface of anhydrous borax is a physical bonding rather than chemical bonding.

After treating the powder surfaces with SA via the dry coating, the presence of carbon element in the XPS analysis, which is the primary indicator of stearic acid in the investigation of the surface chemistry of ANB samples, was evaluated. XPS wide-scan spectra of the raw and surface-coated ANB powders indicate the spectrums are dominated by $\mathrm{B}, \mathrm{Na}$, and $\mathrm{O}$ signals. Additionally, a strong $\mathrm{C} 1 \mathrm{~s}$ peak was detected in SAcoated ANB powders, while a weak C1s signal, owing to likely due to carbonaceous contamination, and was observed in the raw ANB powders. The strong C1s peak confirmed the presence of the stearic acid on the surface of ANB powders.

It has been found that surface coated and uncoated ANB powders exhibit a similar thermal behaviour at the comparatively displayed DTA curves. However, there is a broad but slight hump-shaped curve, appeared at temperatures range between $300-500{ }^{\circ} \mathrm{C}$, and this can speculate to be associated with the degradation of stearic acid. The boiling temperature of stearic acid $\left(383^{\circ} \mathrm{C}\right)$, appears to correspond to the maximum point of the mentioned hump-shaped curve. These observations are comparable with those of Heller-Kallai et al. [44]. The TGA analysis results suggest that stearic acid was bound to the surface of the anhydrous borax by a physical mechanism rather than chemical bonding, due to the fact that stearic SA coating caused only a change in mass.

When the effect of stearic acid coating on the prevention of hydration of ANB powders is examined by XRD analysis, it is seen that the pristine ANB powders are transformed into a form containing 10 moles of water after washing treatment. On the other hand, SA-coated ANB powders are transformed into a form containing highly 5 moles of water instead of 10 moles. The phenomenon is caused by the hydrophobic nature of the SA coating that acts as a barrier delaying the hydration of ANB. This result suggests that stearic acid was bound to the surface of the anhydrous borax because of its significantly preventing further hydration. Admittedly, the expectation is to prevent the hydration completely by the produced coating. However, as observed in the SEM analysis, the discrete coating significantly reduced the intensity of the hydration, although it could not entirely prevent hydration.

When the rheological behaviours of suspensions containing surface-coated and uncoated ANB powders were compared, reductions in the viscosity value and thixotropic hysteresis loop width were interpreted as the positive effect of the dry coating. Solubility and ion release of borates in aqueous suspensions negatively affects the dispersion stability [11], as mentioned before. However, SA coating that acts as a barrier delaying hydration of ANB prevented further ion release by reducing dissolution. Thus, it enhanced the stability of ANB powders in suspension. Finally, it has been determined that the suspensions containing ANB coated with SA have a better stabilisation compared with the suspensions containing raw ANB, regardless of the dispersant type and amount.

Compared with the results in the dry coating study with magnesium stearate [36], it showed that the stearic acid $(8.6 \%)$ to reduce the water solubility of anhydrous borax would be better as a coating agent than magnesium stearate (10\%). This solubility value was achieved with $1 \mathrm{wt} \%$ magnesium stearate and after 120 minutes of dry coating, while lower solubility values were achieved with the same amount of stearic acid and much shorter processing time (60 min). As a result, it has been observed that lower solubility values at the shorter processing times could be achieved with stearic acid than that of magnesium stearate.

\section{Conclusions}

This study presents structural changes concerning the dry coating of anhydrous borax powders by means of stearic acid addition in a planetary mill observing the changes promoted by varying modifier agent amount and processing period. Anhydrous borax is a water-soluble powder and could be altered to the poorly soluble 
state via dry coating with stearic acid. The water solubility of anhydrous borax decreased from $40 \%$ to $8.6 \%$, and a coating efficiency of approximately $68 \%$ was achieved with the stearic acid amount of $1 \mathrm{wt} \%$ at a coating period of $60 \mathrm{~min}$. In our previous study with $\mathrm{Mg}-\mathrm{St}$, the solubility of ANB powders could be reduced to $10.5 \%$ after coating 120 min processing time. In this study, lower solubility values (8.6\%) were obtained by a shorter time coating process with SA, which is another type of fatty acid and is cheaper than Mg-St.

Anhydrous borax surfaces are also highly hydrophilic $\left(17^{\circ}\right)$, and their surface wettability was altered to hydrophobic $\left(99^{\circ}\right)$ via dry coating with stearic acid. DTATG, FT-IR and XPS results indicated that stearic acid is coated onto the anhydrous borax surface via physical adsorption rather than chemical bonding. SEM analysis results also demonstrate that stearic acid is discretely coated onto the anhydrous borax surface. This discrete coating of stearic acid onto the surface of the anhydrous borax significantly prevented its further hydration. In conclusion, an efficient dry coating process by a one-step coating approach could be applied to obtain a modified anhydrous borax surface, which offers controlled physical behaviour in water-based suspensions. The results obtained avoiding the hydration of anhydrous borax by this surface protection are advantageous for a wide range of water-based applications such as paints, drugs, glazes, or ceramics.

\section{References}

[1] McMillian P.W., Glass-Ceramics, 2nd edition, Academic Press, New York, 1979.

[2] Akbay E., Altiokka M. R., Kinetics of borax dehydration by thermal analysis, Anadolu Univ. J. Sci. Technol. AAppl. Sci. Eng. 18 (3), 713-719, 2017.

[3] Kocakuşak S., Akcay K., Ayok T., Tolun R., Production of anhydrous, crystalline borax in a fluidized bed, Ind. Eng. Chem. Res. 35 (4), 1996.

[4] Eti Mine Enterprises, Boron products technical data sheet (in Turkish), Turkey, 1-107, 2018.

[5] Derluyn H., Moonen P., Carmeliet J., Numerical modeling of crystallization induced damage processes, Workshop CRYSPOM III, Crystallization in porous media, Portugal, 2012.

[6] Cooke R. U., Smalley I. J., Salt weathering in deserts, Nature, 220, 1226-1227, 1968.

[7] Flatt R. J., Caruso F., Sanchez A. M. A., Scherer G. W., Chemo-mechanics of salt damage in stone, Nat. Commun., 5, 4823, 2014

[8] Charola A. E., Weber J., The hydration-dehydration mechanism of sodium sulphate, In: 7th International Congress on Deterioration and Conservation of Stone, Proc. Lisbon: LNEC, 581-590, 1992.

[9] Rodriguez-Navarro C., Doehne E., Sebastian E., How does sodium sulphate crystallize? Implications for the decay and testing of building materials, Cem. Concr. Res., 30 (10), 1527-1534, 2000.
[10] Gomez-Tena M. P., Moreno A., Bou E., Cook S., Galindo M., Vicente M. J., Use of a new borate raw material for glaze formulation, Bol. Soc. Esp. Ceram. Vidr. 49 (4), 319-326, 2010.

[11] Kaplan J., Zamek J., A substitute for gerstley borate, Ceram. Tech., 24-29, 2011.

[12] Haber R. A., Powders: Prefiring, in Concise encyclopedia of advanced ceramic materials, ed. R. J. Brook, Pergamon Press, pp. 377-380, 1991.

[13] Ouabbas Y., Dodds J., Galet L., Chamayou A., Baron M., Particle-particle coating in a cyclomix impact mixer, Powder Technol. 189, 245-252, 2009.

[14] Mujumdar A., Wei D., Dave R. N., Pfeffer R., Wu C. $\mathrm{Y}$., Improvement of humidity resistance of magnesium powder using dry particle coating, Powder Technol., 140, 86-97, 2004.

[15] Saleh K., Guigon P., Coating and Encapsulation Processes in Powder Technology, Elsevier, 1st edition, Handbook of Powder Technol. vol. 11, ch. 7, 323-375, 2007.

[16] Otles M. S., Modification of surface properties of biopowders by dry particle coating. PhD Thesis, Université de Toulouse, Toulouse, 2008.

[17] Pfeffer R., Davé R. N., Wei D., Ramlakhan M., Synthesis of engineered particulates with tailored properties using dry particle coating, Powder Technol., 117, 40-67, 2001.

[18] Hersey J. A., Ordered mixing: A new concept in powder mixing practice, Powder Technol., 11 (1), 41-44, 1975.

[19] Hudon S., Lapointe-Garant P. P., Simard J. S., Pichieri A., Hammond S., Sienkiewicz G., Abatzoglou N., et al., Evaluation of a dry coating technology as a substitute for roller compaction for dry agglomeration applications in the pharmaceutical industry, J. Pharm. Innov., $1-18,2018$.

[20] Cavaillès F., Sescousse R., Chamayou A., Galet L., Production of composite particles using an innovative continuous dry coating process derived from extrusion, Advanced Powder Technol., 28 (11), 2875-2885, 2017.

[21] Singh P., Solanky T. K. S., Mudryy R., Pfeffer R., Dave R. N., Estimation of coating time in the magnetically assisted impaction coating process, Powder Technol. $121,159-167,2001$

[22] Yang J., Sliva A., Banerjee A., Dave R. N., Pfeffer R., Dry particle coating for improving the flowability of cohesive powders, Powder Technol. 158, 21-33, 2005.

[23] Sonoda R., Horibe M., Oshima T., Iwasaki T., Watano S., Improvement of dissolution property of poorly water-soluble drug by novel dry coating method using planetary ball mill, Chem. Pharm. Bull. 56 (9), 12431247, 2008

[24] Tsai W. T., Microstructural characterization of calcitebased powder materials prepared by planetary ball milling, Materials, 6, 3361-3372, 2013.

[25] Suryanarayana C., Mechanical alloying and milling, Prog. Mater Sci., 46, 1-184, 2001.

[26] Baláž P., Mechanochemistry in Nanoscience and Min- 
erals Engineering, Chapter 2, High Energy Milling, Springer, Hardcover, Netherland, 2008.

[27] Domka L., Modification estimate of kaolin, chalk, and precipitated calcium carbonate as plastomer and elastomer fillers, Colloid Polym. Sci., 272, 1190-1202, 1994.

[28] Gilbert M., Petiraksakul P., Mathieson I., Characterisation of stearate-stearic acid coated fillers, Mater. Sci. Technol., 17, 1472-1478, 2001.

[29] Wang Y., Eli W., Zhang L., Gao H., Liu Y., Li P., A new method for surface modification of nano-CaCO3 and nano-Al2O3 at room temperature, Adv. Powder Technol., 21 (2), pp. 203-205, 2010.

[30] Mihajlovic S. R., Vucinic D. R., Sekulic Z. T., Milicevic S. Z., Kolonja B. M., Mechanism of stearic acid adsorption to calcite, Powder Technol., 245, 208-216, 2013.

[31] Gilbert M., Sutherland I., Guest A., Characterization of coated particulate fillers, J. Mater. Sci., 35 391-397, 2000.

[32] Markley K.S., Fatly Acids: Their Chemistry and Physical Properties, Part 1, 2nd edition, Interscience Publishers Inc., New York, 1960.

[33] Agernäs O., Tengberg T., Development of Two Methods to Evaluate Lubricating Greases Using a Rheometer BSc, Chalmers University of Technology, Göteborg, Sweden, 2011.

[34] Zhou X., Fan Z., Jin D., Qiu H., Yang J., Comparative study of the modification of mineral powder by titanate and stearic acid and preliminary investigation for their mechanism, J. Mater. Appl., 2 (1), 29-32, 2013.

[35] Liao J., Du G., Qiao X., Hao D., Surface modification of diatomite by stearic acid and its effects on reinforcing for natural rubber/styrene-butadiene rubber blend, J. Chin. Ceram. Soc., 39, 641-645, 2011.
[36] Akpinar S., Yazici Z. O., Can M. F., Investigation of surface-modified anhydrous borax utilisation in raw glazes, Ceram. Int. 44, 18344-18351, 2018.

[37] Koskela J., Effect of mechanical dry coating on powder properties-microcrystalline cellulose and magnesium stearate, Msc. Thesis, University of Helsinki, Division of Pharmaceutical Chemistry and Technology, Helsinki, 2015.

[38] Hong S. H., Lee D. W., Kim B. K., Manufacturing of aluminum flake powder from foil scrap by dry ball milling process, J. Mater. Process. Technol. 100 (1), 105-109, 2000.

[39] Babuccuoglu Y., Reinforcement of Epoxies by Boron Minerals PhD Thesis, Middle East Technical University, Polymer Science and Technology Department, Ankara, 2015.

[40] Pal M., Roy B., Pal M., Structural characterization of borate glasses containing zinc and manganese oxides, J. Mod. Phys., 2, 1062-1066, 2011.

[41] Focke W. W., Molefe D., Labuschagne F. J. W., Ramjee $S$., The influence of stearic acid coating on the properties of magnesium hydroxide, hydromagnesite and hydrotalcite powders, J. Mater. Appl., 44 (22), 6100-6109, 2009

[42] Qing Y., Yang C., Sun Y., Zheng Y., Shang Y., Liu C., Simple method for preparing $\mathrm{ZnO}$ superhydrophobic surfaces with micro/nano roughness, J. Adhes. Sci. Technol., 29 (20) 2153-2159, 2015.

[43] Waclawska I., Thermal decomposition of borax, J. Therm. Anal., 43 (1), 261-269, 1995.

[44] Heller-Kallai L., Yariv S., Friedman I., Thermal analysis of the interaction between stearic acid and pyrophillite or talc. IR and DTA studies, J. Therm. Anal., 31, 95106, 1986. 